\title{
Development of a combined XRF/XPS surface-analysis system for the surface-layer quantification of ${ }^{28} \mathrm{Si}$ spheres
}

\author{
Yu-Hsin $\mathrm{Wu}^{1}{ }^{1}$, Lin Tsao ${ }^{1}$, Jeng-Yu Chiu ${ }^{1}$, Sheng-Jui Chen ${ }^{1}$, Michael Kolbe ${ }^{2}$, Rolf Fliegauf ${ }^{2}$, Edyta Beyer ${ }^{3}$, \\ Frank Haertig ${ }^{3}$ \\ ${ }^{1}$ Center for Measurement Standards, Industrial Technology Research Institute, 30011 Hsinchu, Taiwan \\ 2 Physikalisch-Technische Bundesanstalt (PTB), 10587 Berlin, Germany \\ 3 Physikalisch-Technische Bundesanstalt (PTB), 38116 Braunschweig, Germany
}

\begin{abstract}
To achieve a new kilogram definition using the X-ray crystal density method, the Center for Measurement Standards, Industrial Technology Research Institute in Taiwan has established the combined XRF (X-ray fluorescence)/XPS (X-ray photoelectron spectroscopy) surface analysis system for the quantitative surface-layer analysis of Si spheres. The surface layer of a Si sphere is composed primarily of an oxide layer, carbonaceous contamination and physisorbed/chemisorbed water. This newly combined instrument has been implemented to measure the XRF for the direct determination of the mass deposition of oxygen $\left(\mathrm{ng} / \mathrm{cm}^{2}\right)$ with a calibrated silicon drift detector and the XPS for the ratio between the elements $(\mathrm{O}, \mathrm{Si}, \mathrm{C})$ and composition identification. These two complementary methods of X-ray metrology allow an accurate determination of the surface-layer mass of the Si sphere. In this paper, the construction of a combined XRF/XPS surface-analysis system is reported, including the surface characterisation method, the assembly of parts of the loadlock chamber and ultra-high-vacuum analysis chamber, the vacuum-system design, hardware integration and the intended research on surface-layer measurement. It is anticipated that the measured surface-layer mass will be combined with the core mass of the Si sphere.
\end{abstract}

\section{Section: RESEARCH PAPER}

Keywords: XRCD; Si sphere; XPS; XRF

Citation: Yu-Hsin Wu, Lin Tsao, Jeng-Yu Chiu, Sheng-Jui Chen, Michael Kolbe, Rolf Fliegauf, Edyta Beyer, Frank Haertig, Development of a combined XRF/XPS surface-analysis system for the surface-layer quantification of 28Si spheres, Acta IMEKO, vol. 10, no. 1, article 39, March 2021, identifier: IMEKO-ACTA10 (2021)-01-39

Editor: Koji Ogushi, NMIJ, Japan

Received May 15, 2020; In final form August 5, 2020; Published March 2021

Copyright: This is an open-access article distributed under the terms of the Creative Commons Attribution 3.0 License, which permits unrestricted use, distribution, and reproduction in any medium, provided the original author and source are credited.

Funding: This work is supported by the Bureau of Standards, Metrology and Inspection (BSMI), Taiwan, Republic of China

Corresponding author: Yu-Hsin Wu, e-mail: YH.Wu@itri.org.tw

\section{INTRODUCTION}

At the $26^{\text {th }}$ meeting of the Conférence générale des poids et mesures (CGPM) in 2018 [1], the definition of the unit of mass, the kilogram, was redefined by a fixed value of the Planck constant $h=6.62607015 \times 10^{-34} \mathrm{~J}$ s to replace the International Prototype of the Kilogram (IPK), and the new definition came into force on 20 May 2019 [2], [3]. There are two primary methods for realising the definition of the kilogram, the Kibble balance, which compares electrical power to mechanical power [4]-[6] and the X-ray-crystal-density (XRCD) method [7]-[10]. With the transmission of the information and technology from Physikalisch-Technische Bundesanstalt (PTB, Germany), the Center for Measurement Standards, Industrial Technology Research Institute in Taiwan (CMS/ITRI) adopted the XRCD method to realise the new kilogram definition and established the combined X-ray fluorescence $(\mathrm{XRF}) / \mathrm{X}$-ray photoelectron spectroscopy (XPS) surface-analysis system to evaluate the surface-layer mass of a Si sphere. In this paper, we report the surface-characterisation method and current progress, which includes the system assembly, vacuum-chamber design, hardware integration and the intended work on the construction of the XRF/XPS surface-analysis system.

\section{THE SURFACE-LAYER COMPOSITION OF THE ${ }^{28} \mathrm{SI}$ - ENRICHED SPHERE}

Based on the new Plank constant, XRCD will be used to determine the mass of the Si sphere to realise the new kilogram. The core mass $m_{\text {core }}$ of the Si sphere is determined by counting the number of $\mathrm{Si}$ atoms inside a ${ }^{28} \mathrm{Si}$-enriched sphere and 


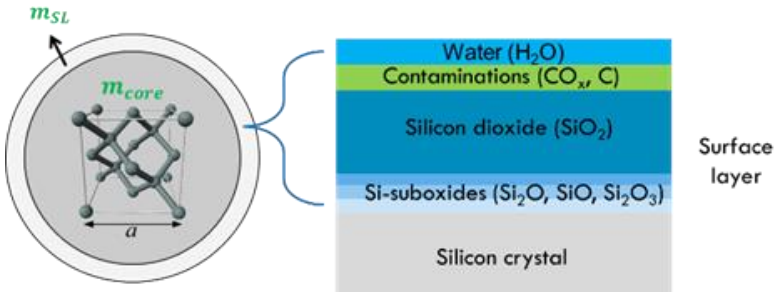

Figure 1. Model of the surface layer of the Si sphere.

multiplying it by the Si mean molar mass [11], [12]. However, an oxide layer and carbonaceous layer is formed on the sphere surface. The mass of the Si sphere $m_{\text {sphere }}$ should take the mass correction of the surface layer into account:

$$
m_{\text {sphere }}=m_{\text {core }}+m_{\mathrm{SL}} \text {. }
$$

The surface-layer (SL) model (Figure 1) for the Si sphere is composed of an oxide layer with $\mathrm{SiO}_{2}$ and suboxides $\left(\mathrm{Si}_{2} \mathrm{O}, \mathrm{SiO}\right.$, $\left.\mathrm{Si}_{2} \mathrm{O}_{3}\right)$ at the interface between the silicon crystal and carbonaceous contaminations $\left(\mathrm{CO}_{\mathrm{x}}, \mathrm{C}\right.$, hydrocarbon) as well as the physisorbed/chemisorbed water layer [13]-[15]. To identify the chemical elements and determine the mass present in the surface, an accurate evaluation of surface layers by XPS and XRF spectrometry is conducted [16].

\section{SURFACE CHARACTERISATION}

\subsection{Combined XRF/XPS analysis for a surface layer}

The quantitative surface analysis of the silicon sphere combines the XRF and XPS measurement techniques. XRF is used to quantify the mass deposition of oxygen on the Si-sphere surface layer based on the calibrated $\mathrm{SiO}_{2}$ reference samples, and the XPS measurement provides information on the chemical state in the oxide and carbonaceous layers, which can be used for a surface analysis of the composition by stoichiometry. With the development of the combined XRF/XPS surface-analysis system, both the XRF and XPS measurements can be carried out using this integrated instrument.

\subsection{X-ray fluorescence}

XRF is a typical method of elemental and chemical analysis. The characteristic X-ray is emitted from a material that has been excited by being bombarded with high-energy X-rays or gamma rays (Figure 2).

For Si spheres, XRF is utilised to measure the surface density, i.e. the mass deposition $\left(\mathrm{ng} / \mathrm{cm}^{2}\right)$, of the oxygen present in the surface layer. Since the in-house X-ray source is not stable enough for element quantification, a synchrotron radiationbased reference-free X-ray fluorescence analysis [17]-[20] is

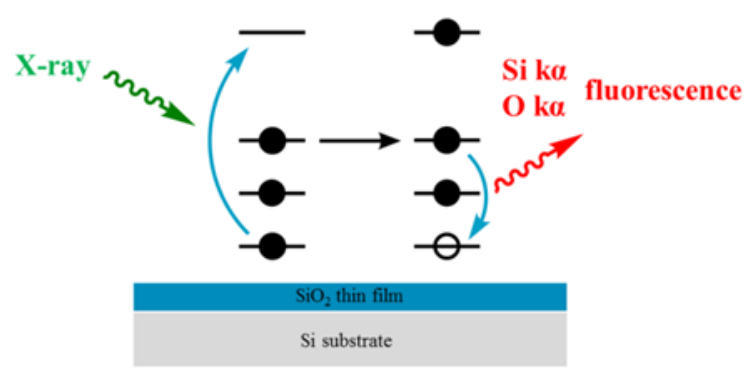

Figure 2. Schematic diagram of X-ray fluorescence from an $\mathrm{SiO}_{2}$ thin film with a thickness of several nanometres.

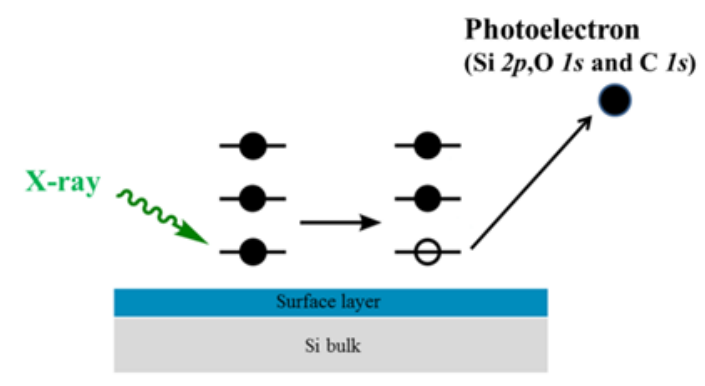

Figure 3. Schematic diagram shows the photoelectrons escaping from the surface layer of a Si sphere.

employed for the direct determination of the mass deposition of oxygen. With this technique, the mass deposition of oxygen from five $\mathrm{SiO}_{2}$ reference samples with a nominal thickness ranging from $2 \mathrm{~nm}$ to $10 \mathrm{~nm}$ are measured at BESSY II, Berlin. The combined XRF/XPS surface-analysis system is used to measure the fluorescence intensities of oxygen and silicon to obtain the intensity ratio between $\mathrm{O} k \alpha(525 \mathrm{eV})$ and $\mathrm{Si} \mathrm{k} \alpha(1740 \mathrm{eV})$ from the reference samples. According to Sherman's equation, the correlation of the mass deposition in relation to its fluorescence radiation of $\mathrm{O} k \alpha /(\mathrm{Si} k \alpha)$ is given as

$$
m d_{O}=-\frac{G}{\mu_{\mathrm{tot}}} \ln \left(1-C_{0} R_{\mathrm{OSi}} \mu_{\mathrm{tot}}\right) \text {, }
$$

where $m d_{0}$ is the mass deposition of oxygen, $C_{0}$ is the calibration factor, $\mu_{\text {tot }}$ is the total mass attenuation coefficient, $G$ is the geometry factor and $R_{o s i}$ is the intensity ratio of $O k \alpha$ to $S i k \alpha$. Most of the $\mathrm{Si} \alpha \alpha$ signal is from the Si substrate. The ratio of $\mathrm{O}$ $k \alpha /(\operatorname{Si~} k \alpha)$ reflects the amount of change in the oxygen. The mass deposition of oxygen of the $\mathrm{Si}$ sphere can be obtained by interpolation using the correlation curve with a ratio of $\mathrm{O} k \alpha /(\mathrm{Si}$ $\mathrm{k} \alpha$ ) measured from the Si sphere.

\subsection{X-ray photoelectron spectroscopy}

XPS is a surface-sensitive measurement technique. The photoelectrons escape from the sample because the X-ray energy irradiating the sample is larger than the binding energy of the elements. The kinetic energy of the photoelectrons is analysed by an electron spectrometer to identify the chemical elements and their binding states (chemical shift), as shown in Figure 3.

Without the reference samples used for XPS measurement, only ratios between $\mathrm{Si} 2 p, \mathrm{O} 1 s$ and $\mathrm{C} 1 s$ are quantified according to the amount of the photoelectrons detected. In the surface layers, the relative atomic fraction $\mathrm{C}_{\mathrm{A}}$ between the elements is obtained by the normalised XPS signal $I_{\mathrm{j}_{\mathrm{A}} \text { : }}^{\prime}$

$$
I^{\prime}{ }_{j A}=\frac{I_{j A}}{\sigma_{j A} \cdot \lambda(\mathrm{KE}) \cdot T(\mathrm{KE})},
$$

where $T(\mathrm{KE})$ is the transmission function, $\sigma_{j \mathrm{~A}}$ is the photoelectric cross section of photoemission $j$ of element $A[21]$ and $\lambda(\mathrm{KE})$ is the inelastic mean free path of the photoelectrons [22]. The atomic fraction can be expressed as follows:

$$
C_{A}=\frac{I_{j A}^{\prime}}{\sum I_{j A}^{\prime}} \text {. }
$$

The ratio between the elements measured by XPS is proportional to the number of atoms of each element. With the mass deposition of oxygen determined by XRF, the number of oxygen atoms per unit area can be obtained; the absolute mass 
depositions of $\mathrm{Si}$ and $\mathrm{C}$ can be calculated by the mass deposition of oxygen in the surface layer based on XRF. The detailed XPS scans of $\mathrm{Si} 2 p$ and $\mathrm{C} 1 s$ give the ratios corresponding to different binding states. For example, there are several peaks in $\mathrm{Si} 2 p$ in the XPS measurement. The main peak is from the silicon bulk, and the subpeaks are from $\mathrm{SiO}_{2}$ and suboxides $\left(\mathrm{Si}_{2} \mathrm{O}, \mathrm{SiO}\right.$ and $\mathrm{Si}_{2} \mathrm{O}_{3}$ ) in the interface. The distribution of the oxygen present in the silicon oxide and carbonaceous layers can be evaluated using a stoichiometric approach [23]. Since hydrogen cannot be detected by XPS, the mass deposition of hydrogen is estimated and addressed by the possible molecules in the carbonaceous layer. For chemisorbed water, the estimation of the mass deposition of hydrogen relies on the remaining oxygen and the silicon-hydroxyl group in the silicon-oxide layer [24].

\section{CONSTRUCTION OF XRF/XPS SURFACE-ANALYSIS SYSTEM}

CMS/ITRI established the combined XRF/XPS surfaceanalysis system in 2019. This system is designed to carry out the XRF and XPS measurement for Si spheres. The construction work includes the assembly of parts of the load-lock chamber, the ultra-high-vacuum (UHV) analysis chamber, hardware integration and the design of the vacuum system.

The combined XRF/XPS surface-analysis system is shown in Figure 4. The system is mainly composed of two chambers, the load-lock chamber and the UHV-analysis chamber. The Si sphere is placed in the load-lock chamber and transferred into the UHV-analysis chamber for the surface-layer measurement. The incident radiation of the characteristic $\mathrm{Al} \mathrm{K} \alpha$ fluorescence line is $1486.6 \mathrm{eV}$ with an energy resolution of $\Delta \mathrm{E}$ of $250 \mathrm{meV}$ by quartz crystal using Rowland geometry. The geometry between the incident radiation and the photoelectron detection channel (electron spectrometer) is fixed at the magic angle of $54.7^{\circ}$, with a pass energy of $80 \mathrm{eV}$ for the spectrum survey and $40 \mathrm{eV}$ for the detailed scan of each element. A Bruker SDD (silicon drift detector) fluorescence detector with a windowless detecting area of $30 \mathrm{~mm}^{2}$ is set up with respect to the incident radiation at angles of $45^{\circ}$ out of plane and $15^{\circ}$ in plane. The key component of this analysis system is the UHV five-axis manipulator to investigate the full Si-sphere surface in different geometries inside the UHV-analysis chamber (Figure 5). For the five-axis manipulator, two of the axes are linear motors moving along the vertical (axis (2)) and horizontal (axis (4)) axes with a step resolution of $0.000061 \mathrm{~mm}$ and $0.0001 \mathrm{~mm}$, respectively, to

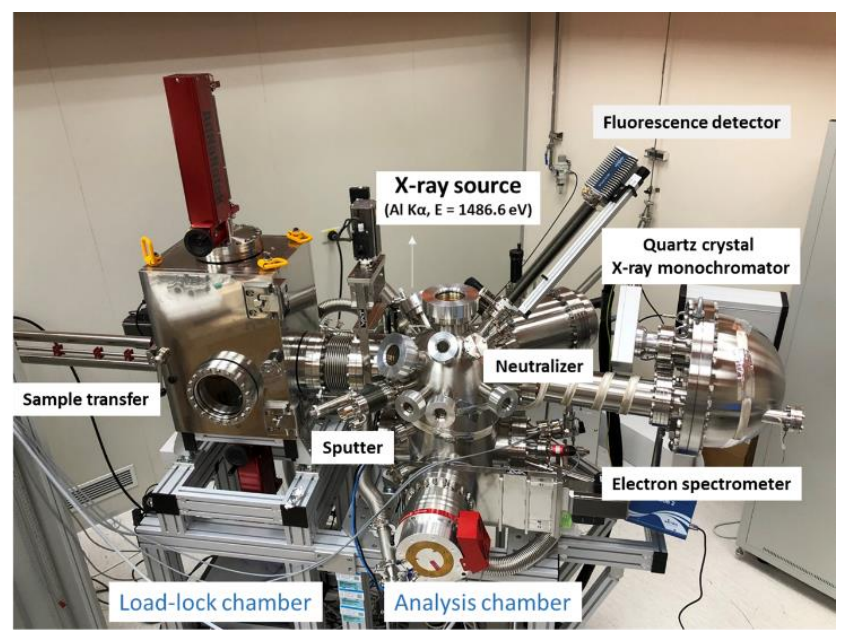

Figure 4. The combined XRF/XPS surface-analysis system.
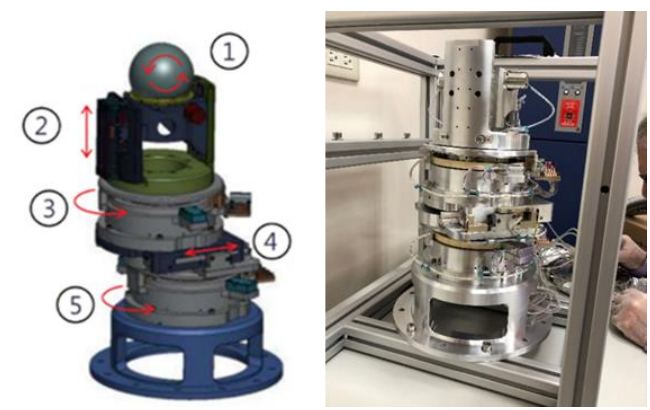

Figure 5. Schematic diagram (left) and the view (right) of the UHV five-axis manipulator. Axes (1) and (3) are rotated around the centre of the Si sphere, axis (5) is rotated around the centre of the chamber and axes (2) and (4) move linearly to adjust the position of the Si sphere.

adjust the position of the Si sphere to the centre of the chamber. The other three axes are composed of rotating motors. The motor at the lowest axis (axis (5)) rotates around the centre of the UHV-analysis chamber with a step resolution of $0.0001^{\circ}$ to change the angle of incidence on the Si sphere. The upper two motors rotate around the centre of the Si sphere to measure element distribution. The step resolutions of horizontal axis (1) and vertical axis (3) are $0.00014^{\circ}$ and $0.0001^{\circ}$, respectively.

When the measurement is underway, the chambers should be maintained in an ultra-high-vacuum environment with the pressure of the load-lock chamber at $10^{-5} \mathrm{~Pa}$ and the UHVanalysis chamber (main chamber) at $10^{-7} \mathrm{~Pa}$. The design of the vacuum system is shown in Figure 6. The load-lock chamber and main chamber are connected by a gate valve, the turbo molecular pumps are mounted on both chambers and connected to a root pump and an ionisation gauge and Pirani gauge are used to monitor the pressure in both chambers. As a buffer to the main chamber, the load-lock chamber should be evacuated and refilled when changing the sample to be measured. An angled valve with a soft pump is used to avoid the diffusion of dust and particles in the load-lock chamber; the load-lock chamber will be filled with nitrogen before being opened. Moreover, in order to avoid the window on the chamber being damaged by the high pressure of the nitrogen, a buffer volume is connected between the source of the nitrogen and the load-lock chamber. To maintain the pressure of the main chamber at $10^{-7} \mathrm{~Pa}$, except when using a turbo molecular pump, an ion pump is also used, and a residual-gas analyser (RGA) is used to analyse the residual pressure in the main chamber.

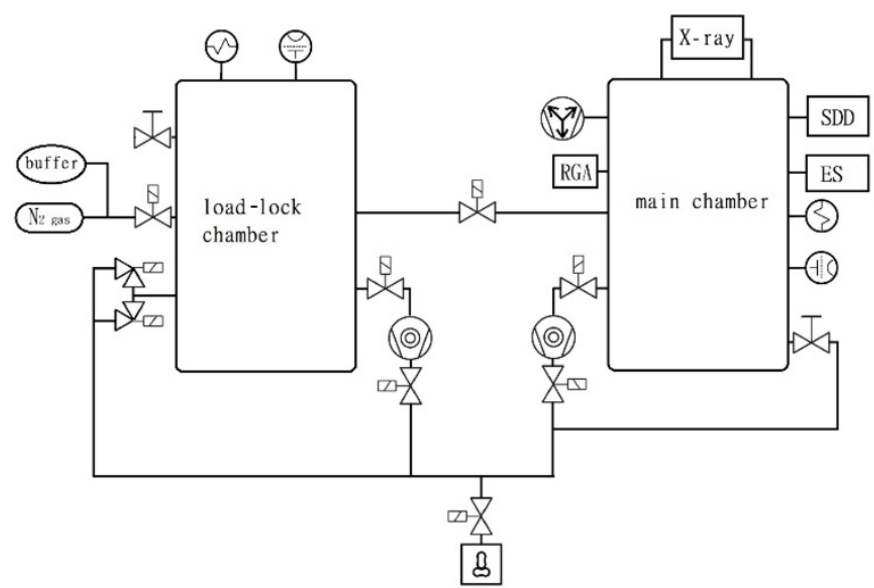

Figure 6. The vacuum-system design of the combined XRF/XPS surfaceanalysis system. 
In addition, for the preparation of the XRF measurement, the response function and detector efficiency of the SDD has been calibrated in the energy range of $100 \mathrm{eV}$ to $1850 \mathrm{eV}$ [25]. The response function of the SDD is measured by changing the energy of the incident beam from monochromatised synchrotron radiation and will be convoluted with the theoretical bremsstrahlung and resonant Raman scattering models [26]. To quantify the mass deposition of oxygen on the five $\mathrm{SiO}_{2}$ reference samples with thicknesses ranging from $2 \mathrm{~nm}$ to $10 \mathrm{~nm}$, the samples were measured in PTB's laboratory at the BESSY II in Berlin for synchrotron radiation. Monochromatised synchrotron radiation at $684 \mathrm{eV}$ photon energy was used to irradiate the sample. With the calibrated photodiode and SDD to collect the signal of the incident beam and fluorescence, the mass deposition of the oxygen can be directly determined.

\section{CONCLUSION AND FUTURE WORK}

In conclusion, CMS/ITRI has completed the construction work on the combined XRF/XPS surface-analysis system, including the assembly of parts of the load-lock chamber, UHVanalysis chamber, vacuum-system design and hardware integration. It is anticipated that the system will measure the surface-layer mass of the $\mathrm{Si}$ sphere periodically to maintain the primary mass standard via the XRCD method. To measure and characterise the surface-layer mass of a Si sphere, any future work can be divided into two phases. In the first phase, the preprocessing work before starting the XRF and XPS measurement of the Si sphere should be completed. For this, the hardware and software control system must be integrated - the light path is adjusted to pass through the centre of the chamber and the sample is aligned for the angle of incidence of the XRF and XPS measurement, respectively. For the XRF measurement, the correlation of the mass deposition measured by PTB will be fitted with the fluorescence radiation of $\mathrm{O} \mathrm{k} \alpha / \mathrm{Si} \mathrm{k} \alpha$ by the XRF/XPS surface-analysis system according to eqn (2). For the XPS measurement, as one of the factors is intensity normalisation (eqn (3)), the transmission function T(KE) of the XPS spectrometer must be estimated using the quantified peakarea approach method by measuring Au 4f, Au 4d, Au 4p3/2, Ag

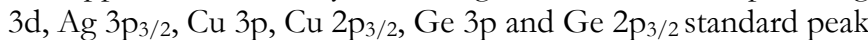
areas from reference samples at a pass energy of $40 \mathrm{eV}$ and 80 $\mathrm{eV}$ [27]. In the second phase, the expected goal is to provide the surface-layer mass and uncertainty of the Si sphere. First, the XPS or XRF measurement should repeat the scan of the Si sphere several times to check for the angular setting liability of the UHV five-axis manipulator. The measured $\mathrm{O} / \mathrm{Si}$ fluorescence intensity of the Si sphere is interpolated by the fitted calibration curve to obtain the mass deposition of oxygen present in the surface layer. The XPS measurement is then carried out on the whole surface of the Si sphere to calculate the ratio of photoelectrons between Si, C and O. The uncertainty of the surface-layer mass will be evaluated from the source for the XRF measurement, including the calibrated mass deposition of the $\mathrm{SiO}_{2}$ reference samples, the fitted $\mathrm{O} / \mathrm{Si}$ ratios and the reproducibility.

To give the mass of the ${ }^{28} \mathrm{Si}$-enriched sphere, the mass of the surface layer measured by the combined XRF/XPS surfaceanalysis system should be monitored for its stability and combined with the mass from the sphere core. However, since the surface-layer measurement is carried out in a vacuum, the sorption correction for evaluating the mass difference of the mass standards transferred between in air and in vacuum have to be considered for mass dissemination [28].

\section{REFERENCES}

[1] Resolution 1 of the 26th CGPM (2018). Online [Accessed 29 March 2021] https://www.bipm.org/en/CGPM/db/26/1/

[2] M. Stock, R. Davis, E. Mirandés, M. J. T. Milton, The revision of the SI-the result of three decades of progress in metrology, Metrologia 56 (2019) 022001.

DOI: $\underline{10.1088 / 1681-7575 / a b 28 a 8}$

[3] D. B. Newell, The CODATA 2017 values of h, e, $k$, and NA for the revision of the SI, Metrologia 55 (2018), pp. L13-L16. DOI: $10.1088 / 1681-7575 /$ aa950a

[4] I. A. Robinson, The watt or Kibble balance: a technique for implementing the new SI definition of the unit of mass, Metrologia 53 (2016), pp. A46-A74 DOI: $10.1088 / 0026-1394 / 53 / 5 / A 46$

[5] G. A. Shaw, Milligram mass metrology using an electrostatic force balance, Metrologia 53 (2016), pp. A86-A94. DOI: $\frac{10.1088 / 0026-1394 / 53 / 5 / A 86}{Z \text { ( }}$

[6] Z. Li, Z. Zhang, Y. Lu, P. Hu, Y. Liu, J. Xu, Y. Bai, T. Zeng, G. Wang, Q. You, The first determination of the Planck constant with the joule balance NIM-2, Metrologia 54 (2017), p. 763. DOI: $10.1088 / 1681-7575 / a a 7 a 65$

[7] B. Andreas, Y. Azuma, G. Bartl, P. Becker, H. Bettin, M. Borys, I. Busch, P. Fuchs, K. Fujii, H. Fujimoto, E. Kessler, M. Krumrey, U. Kuetgens, N. Kuramoto, G. Mana, E. Massa, S. Mizushima, A. Nicolaus, A. Picard, A. Pramann, O. Rienitz, D. Schiel, S. Valkiers, A. Waseda, S. Zakel, Counting the atoms in a $28 \mathrm{Si}$ crystal for a new kilogram definition, Metrologia 48 (2011), pp. S1-S13. DOI: $10.1088 / 0026-1394 / 48 / 2 /$ S01

[8] R. S. Davis, The assumption of the conservation of mass and its implications for present and future definitions of the kilogram and the mole, Metrologia 51 (2014), pp. 169-173. DOI: $10.1088 / 0026-1394 / 51 / 3 / 169$

[9] P. Cladé, Precise determination of the ratio $\mathrm{h} / \mathrm{mu}$ : a way to link microscopic mass to the new kilogram, Metrologia 53 (2016), pp. A75-A82.

DOI: $10.1088 / 0026-1394 / 53 / 5 / A 75$

[10] K. Fujii, H. Bettin, P. Becker, E. Massa, O. Rienitz, A. Pramann, A. Nicolaus, N. Kuramoto, I. Busch, M. Borys, Realization of the kilogram by the XRCD method, Metrologia 53 (2016), pp. A19A45.

DOI: $10.1088 / 0026-1394 / 53 / 5 / A 19$

[11] N. Kuramoto1, S. Mizushima, L. Zhang, K. Fujita, Y. Azuma, A. Kurokawa, S. Okubo, H. Inaba, K. Fujii, Determination of the Avogadro constant by the XRCD method using a ${ }^{28}$ Si-enriched sphere, Metrologia 54 (2017), pp. 716-729.

DOI: $10.1088 / 1681-7575 /$ aa $77 \mathrm{~d} 1$

[12] A. Pramann, O. Rienitz, D. Schiel, J. Schlote, B. Güttler, S. Valkiers, Molar mass of silicon highly enriched in ${ }^{28} \mathrm{Si}$ determined by IDMS, Metrologia 48 (2011), pp. S20-S25.

DOI: $10.1088 / 0026-1394 / 48 / 2 /$ S03

[13] Y. Azuma, P. Barat, G. Bartl, H. Bettin, M. Borys, I. Busch, L. Cibik, G. D'Agostino, K. Fujii, H. Fujimoto, A. Hioki, M. Krumrey, U. Kuetgens, N. Kuramoto, G. Mana, E. Massa, R. Meeß, S. Mizushima, T. Narukawa, A. Nicolaus, A. Pramann, S. A. Rabb, O. Rienitz, C. Sasso, M. Stock, R. D. Vocke, A. Waseda, S. Wundrack, S. Zakel, Improved measurement results for the Avogadro constant using a ${ }^{28}$ Si-enriched crystal, Metrologia 52 (2015), pp. 360-375

DOI: $10.1088 / 0026-1394 / 52 / 2 / 360$

[14] I. Busch, H.-U. Danzebrink, M. Krumrey, M. Borys, H. Bettin, Oxide layer mass determination at the silicon sphere of the Avogadro project, IEEE Trans. Instrum. Meas. 58 (2009), pp. 891-896. DOI: $10.1109 / C P E M .2008 .4574826$ 
[15] I. Busch, Y. Azuma, H. Bettin, L. Cibik, P. Fuchs, K. Fujii, M Krumrey, U. Kuetgens, N. Kuramoto, S. Mizushima, Surface layer determination for the $\mathrm{Si}$ spheres of the Avogadro project, Metrologia 48 (2011), pp. S62-S82. DOI: $10.1088 / 0026-1394 / 48 / 2 /$ S10

[16] M. Müller, B. Beckhoff, E. Beyer, E. Darlatt, R. Fliegauf, G. Ulm, M. Kolbe, Quantitative surface characterization of silicon spheres by combined XRF and XPS analysis for the determination of the Avogadro constant, Metrologia 54 (2017), pp. 653-662. DOI: $10.1109 /$ CPEM.2016.7540797

[17] B. Beckhoff, Reference-free X-ray spectrometry based on metrology using synchrotron radiation, J. Anal. At. Spectrom 23 (2008), pp. 845-853. DOI: $10.1039 / \mathrm{B} 718355 \mathrm{~K}$

[18] M. Kolbe, B. Beckhoff, M. Krumrey, G. Ulm, Thickness determination for $\mathrm{Cu}$ and $\mathrm{Ni}$ nanolayers: comparison of referencefree fundamental-parameter based X-ray fluorescence analysis and X-ray reflectometry, Spectrochimica Acta B 60 (2005), pp. 505510.

DOI: $10.1016 /$ i.sab.2005.03.018

[19] M. Müller, P. Hönicke, B. Detlefs, C. Fleischmann, Characterization of high-k nanolayers by grazing incidence X-ray spectrometry, Materials 7 (2014), pp. 3147-3159. DOI: $10.3390 / \mathrm{ma} 7043147$

[20] G. Bartl, P. Becker, B. Beckhoff, H. Bettin, E. Beyer, M. Borys, I Busch, L. Cibik, G. D’Agostino, E. Darlatt, M. Di Luzio, K. Fujii, H. Fujimoto, K. Fujita, M. Kolbe, M. Krumrey, N. Kuramoto, E. Massa, M. Mecke, S. Mizushima, M. Müller, T. Narukawa, A. Nicolaus, A Pramann, D. Rauch, O. Rienitz, C. P. Sasso, A. Stopic, R. Stosch, A. Waseda, S. Wundrack, L. Zhang, X. W. Zhang, A new ${ }^{28} \mathrm{Si}$ single crystal: counting the atoms for the new kilogram definition, Metrologia 54 (2017), pp. 693-715. DOI: $\underline{10.1088 / 1681-7575 / a a 7820}$
[21] J. H. Scofield, Hartree-Slater subshell photoionization crosssections at 1254 and $1487 \mathrm{eV}$, Journal of Electron Spectroscopy and Related Phenomena 8 (1976), pp. 129-137. DOI: $10.1016 / 0368-2048(76) 80015-1$

[22] S. Tanuma, Calculations of electron inelastic mean free paths (IMFPS). IV. Evaluation of calculated IMFPs and of the predictive IMFP formula TPP-2 for electron energies between 50 and 2000 eV, Surf. Interface Anal. 20 (1993), pp. 77-89. DOI: $\underline{10.1002 / \mathrm{sia} .740200112}$

[23] L. Zhang, N. Kuramoto, Y. Azuma, A. Kurokawa, K. Fujii, Thickness measurement of oxide and carbonaceous layers on a 28Si sphere by XPS, IEEE Trans. Instrum. Meas. 6 (2016), pp. 1297-1303. DOI: $10.1109 /$ TIM.2016.2634678

[24] S. Mizushima, Determination of the amount of gas adsorption on $\mathrm{SiO} 2 / \mathrm{Si}(100)$ surfaces to realize precise mass measurements, Metrologia 41 (2004), pp. 137-144. DOI: $10.1088 / 0026-1394 / 41 / 3 / 005$

[25] F. Scholze, M. Procop, Modelling the response function of energy dispersive X-ray spectrometers with silicon detectors, X-ray Spectrometry 38 (2009), pp. 312-321. DOI: $10.1002 /$ xrs. 1165

[26] M. Müller, B. Beckhoff, G. Ulm, B. Kanngießer, Absolute determination of cross sections for resonant Raman scattering on silicon, Phys Rev A 74 (2006) 012702. DOI: $10.1103 /$ PhysRevA.74.012702

[27] R. Hesse, Improved accuracy of quantitative XPS analysis using predetermined spectrometer transmission functions with UNIFIT 2004, Surf. Interface Anal. 37 (2005), pp. 589-607. DOI: $10.1002 /$ sia.2056

[28] J. Berry, S. Davidson, Effect of pressure on the sorption correction to stainless steel, platinum/iridium and silicon mass artefacts, Metrologia 51 (2014), pp. S107-S113. DOI: $\underline{10.1088 / 0026-1394 / 51 / 2 / \text { S107 }}$ 